ORIGINAL ARTICLE

\title{
General practice critical incident reviews of patient suicides: benefits, barriers, costs, and family participation
}

\author{
E King, K Kendall, R Wiles, H Rosenvinge, C Gould, A Kendrick
}

See end of article for

authors' affiliations

Qual Saf Health Care 2005;14:18-25. doi: 10.1136/qshc.2003.007344

...................

Correspondence to: Dr E King, Treatment Decisions Group, Community Clinical Sciences Research Division, University Department, Department of Psychiatry, RSH Hospital, Southampton, SOI 4 OYG, UK; E.A.King@soton.ac.uk

Accepted for publication 29 October 2004

\begin{abstract}
Aim: To explore the feasibility of holding critical incident reviews (CIRs) after patient suicides in general practice and their ability to change practice.

Methods: Thirteen practices were invited to conduct a facilitated CIR on 18 current patient suicides. Next of kin views were sought. All staff attending a CIR were interviewed after the review.

Results: Ten practices reviewed 12 deaths. Twenty six staff attended reviews; all were interviewed. Next of kin contributed to six reviews; only one criticised care. Changes following the reviews included steps to improve internal communication and bereavement support to set up internal CIRs and review prescribing policies. Communications between practices and other agencies were clarified.

Conclusion: Practices were willing to hold CIRs and appreciated the potential positive value but need reassurance that they will not be blamed for suicides, and that the cost in time and resources will be recognised.
\end{abstract}

$\mathrm{P}$ revention of suicide is a universal public health issue. ${ }^{1}$ In the UK, suicide accounts for $1 \%$ of all deaths. While not all suicides can or will ever be prevented, the World Health Organisation (WHO $)^{2}$ states that "most suicides can be prevented". An important element of the WHO's strategy to reduce the number of suicides involves training workers in primary care and other sectors to increase awareness about suicidal behaviours and their effective prevention. Primary health care staff in the community are regarded as able to play an important step in suicide prevention" ${ }^{2}$ since "they have a long and close contact in the community and are well accepted by local people and provide the vital link between the community and the health care system. In short they are available, accessible, knowledgeable, and committed to providing continuity of healthcare to the local community". ${ }^{\prime}$

Patient suicides can be devastating for both clinicians and families, arousing feelings of guilt, fear and professional inadequacy in the former and of recrimination in the latter. ${ }^{3}$ It has been estimated that each suicide has a serious impact on at least six other people who may include primary health care staff. ${ }^{2}$ Although the sudden deaths of patients under the care of mental health professionals have been subject to review for some time, ${ }^{45}$ the routine and formal auditing of such deaths in general practice is less common. General practitioners (GPs) often discuss patient suicides informally within the practice, but Standard Seven of the (UK) Mental Health National Service Framework ${ }^{6}$ implies that a more formal audit will be required in the future. Methods developed for turning informal practice discussions into a structured and effective form of audit in primary health care settings have been described as "critical event auditing", "significant event auditing", 8 or the "critical incident technique". 910

A primary health care team using the critical incident technique with the aid of a facilitator to analyse events surrounding patient deaths (from any cause) found the procedure acceptable and felt it provided a useful opportunity for reflecting on their role in patient care. ${ }^{7}$ The extension of the facilitated critical incident technique to the more sensitive audit of suicides ${ }^{11}$ showed that, although the technique might have a limited role in improving the management of suicide risk, a potential strength is to enable reflection on practice in a difficult emotional area. These studies show that such methods can be an effective method of quality assurance, ${ }^{12}$ but rely on a positive team culture.

This study was undertaken to test the feasibility of holding critical incident reviews (CIRs) after suicides in general practice and to identify the barriers to-and benefits ofundertaking CIRs after patient suicides.

The specific questions addressed were:

- are practices willing to audit patient suicides?

- are next of kin willing to contribute?

- do CIRs lead to changes in practice?

- how much do CIRs cost?

- do GPs and primary care team members value the review process?

\section{METHODS}

Ethical approval was granted by Southampton and South West Hants joint research ethics committee. The Southampton Primary Care Trust Board also granted approval.

The study comprised four elements: collection of information for CIRs (from coroners, GP practices and next of kin); holding CIRs; post-review interviews with review participants; and assessment of the impact of reviews (changes in practice) and cost. All those who died by suicide and who were registered with one of the 49 practices in three primary care groups in Southampton and whose inquest was held in a 10 month period were eligible for a CIR (box 1). Two local Coroners provided details of all suicides and open verdict deaths from similar causes after the inquest procedure was completed (box 2). Information was supplied retrospectively for the months May-July 2001 and prospectively for the months August 2001-February 2002, and included name of GP and next of kin. Twenty two inquest files were inspected. 


\section{Box 1 Setting of study}

Patients eligible for a CIR were: (1) subject to an inquest at which a verdict of suicide or an open verdict on a death from a similar cause was returned between August 2000 and March 2001; and (2) registered with one of 190 GPs in 49 practices in three primary care groups of GPs in southern England.

Number of GPs/practice ranged from 1 to 8 (mean 4).

Catchment area of practices fell within two Coroners' districts.

Immediately following the inquest the facilitator invited the practice with which the patient was registered to conduct a CIR. The procedure is described in fig l. GPs accepting the invitation were encouraged to invite all members of the primary care team to the review, irrespective of whether they had direct knowledge of the patient.

\section{Collection of information for the CIR}

Inquest files were inspected and GPs were asked to complete a pre-CIR questionnaire (Appendix 1) and return it to the facilitator before the CIR meeting. GPs were also asked to invite the patient's next of kin to talk about their relative's care during an interview with an independent researcher so that a summary of their views could be considered during the CIR meeting.

If relatives were willing, interviews lasting between 30 and 90 minutes were conducted in the interviewee's home by an experienced researcher (RW). The aim of these interviews was to identify what, if any, issues relating to the care their relative had received prior to their death they wanted fed back to the GP practice. An in-depth interview was considered essential so that relatives could express their views and experiences in relation to this and enable them to identify relevant issues. Audiotaping of these interviews was deemed unnecessary, given their aim was to identify issues to feed back to the GP practice as part of the review rather than to identify and analyse the experience of loss through suicide. Thus, notes were taken during the semi-structured interview designed to include the following topics: the health of and the health care received by their relative; whether they thought the death could have been prevented; and if there were any specific issues they wanted raised at the review. This format enabled the family to talk about their experience of the death, the events leading up to it, and the support that their relative had received. Issues raised in the interview that the family wanted to be considered at the review were summarised on a form which was signed by the interviewee. Any other information discussed in the interview was confidential within the research team and was not subject to further analysis.

At the end of the interview the researcher gave each interviewee a leaflet about a local bereavement service from which they could obtain support if there were issues raised in the interview that had distressed them. The research team agreed that attendance at the review meeting by family members was not appropriate because this might be perceived as threatening to the primary care teams.

\section{CIR meeting}

Following the next of kin interview, or where the next of kin declined to be interviewed, the CIR meeting was held. Attendees included the GP, all invited primary health care staff, the facilitator (HR), and two other members of the research team (EK and CG). CIR meetings lasted for 3080 minutes (average 60 minutes).

\section{Box 2 Coroner's procedure in UK}

All violent, unnatural, or sudden deaths for which the cause cannot be certified by a doctor must be reported to the Coroner in whose district the death is certified and a postmortem examination performed. If the post-mortem examination shows death was not due to natural causes, a public inquest must be held to determine:

- the identity of the deceased;

- the time and location of the event leading to death;

- the medical cause of death (from post-mortem examination); and

- the mode of death.

The inquest verdict summarises the mode of death and reflects the identity and motivation of the perpetrator of the fatal act:

- Suicide (fatal act deliberately self-inflicted).

- Accident (fatal act not deliberately inflicted by either self or another).

- Open verdict (undetermined whether fatal act inflicted by self or another or whether deliberately or accidentally self-inflicted).

The facilitator initiated the discussion based on the pre-CIR questionnaire (Appendix 1). Additional information from the inquest file was made available, as was any response from the next of kin. Practice members discussed potential risk factors and treatment with reference to the patient's notes, practice computer, and inquest file data that included post-mortem drug/alcohol levels and circumstances of death. The facilitator explored whether any practice discussion of the death had occurred, whether relatives had been contacted since the death, the GP's personal reaction to the suicide, and whether intra-practice support was available. The practice team discussed what lessons could be learnt from the suicide in terms of improvements in practice.

The CIR meetings were not recorded but observational notes were made by one of the researchers (CG). The observational data recorded on a pro-forma included: professions of the attending healthcare staff, seating arrangement, sources of information accessed during the review, and the length of meeting. Also noted were the contributions made by each attendee, their body language and perceived comfort with the review. These data were incorporated into the analysis of post-CIR interviews.

\section{Post-CIR interviews}

All practice members who attended a CIR were interviewed after the review. Semi-structured in-depth interviews were conducted to explore the following areas relating to the CIRs: expectations and concerns; barriers and benefits; limitations and problems; future involvement; and recommendations. This approach was chosen because it enabled the interviewer to explore the experiences and perceptions of people about potentially complex and sensitive issues while allowing for the clarification of issues and probing of further information. The interviews, which typically lasted one hour, were audiotaped and transcribed. Field notes were kept and memoranda written to aid data analysis throughout the project. The transcripts were analysed systematically by a process of constant comparison to identify themes. In an iterative process, analysis of each transcript informed future interviews. Similarly, emerging themes were reported at team 


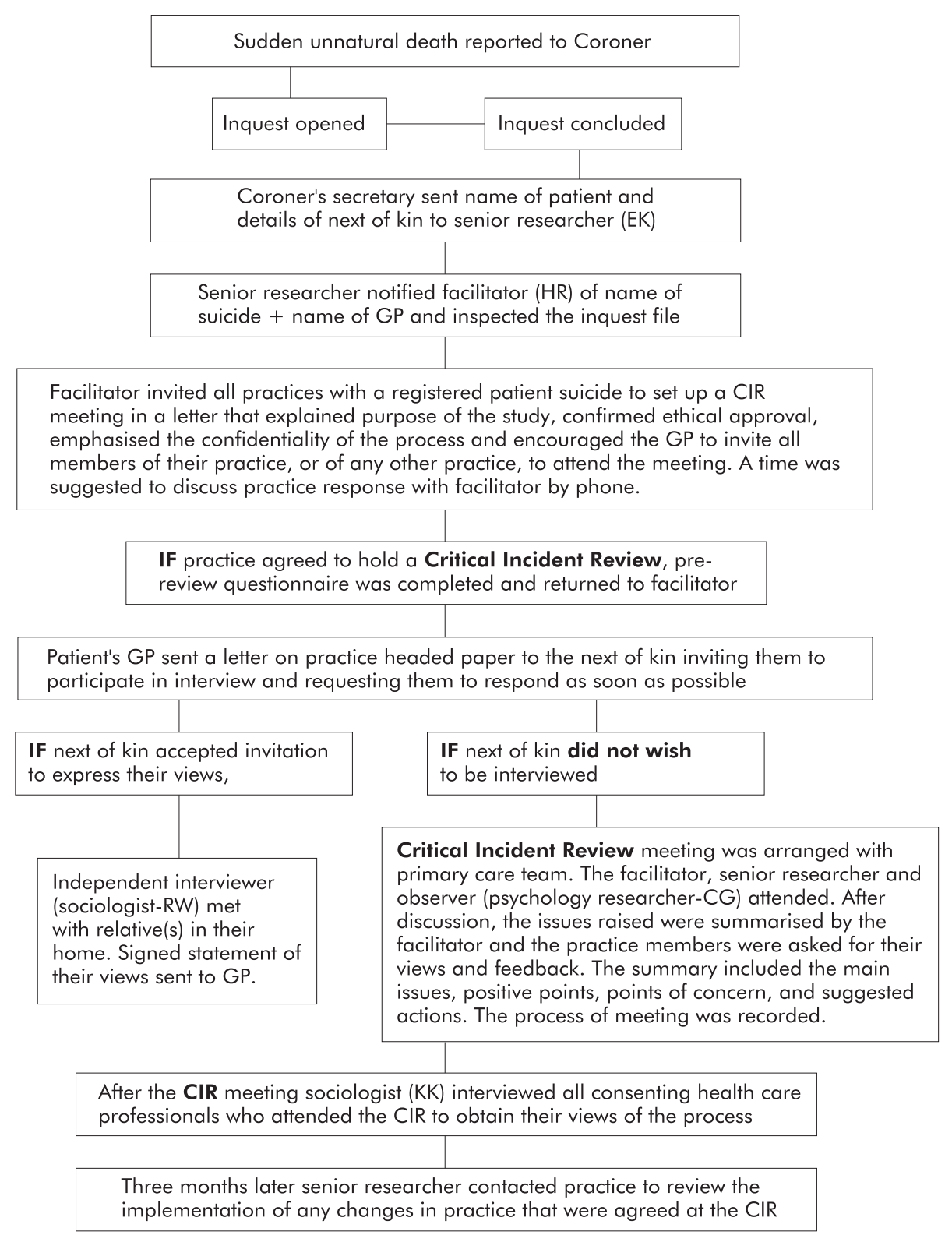

Figure 1 Procedure for initiating/implementing critical incident review (CIR) of suicide in general practice.

meetings of the steering committee where ensuing discussions helped to clarify key themes and inform future interviews. ${ }^{13}$

\section{Impact of reviews}

Three months after the review each CIR practice was contacted to ascertain what actions had been taken concerning the identified issues. The cost of each review in terms of staff time was estimated.

\section{RESULTS}

\section{Were practices willing to audit patient suicides?}

During the 10 month study period, 13 of the 49 practices in the Southampton primary care groups experienced at least one patient suicide (five experienced two suicides). The 18 patients were registered with 16 GPs (fig 2 and box 3). GPs in 10 of the 13 practices agreed to review the deaths of 12 patients. Two of the practices with multiple suicides reviewed both deaths, two reviewed one death, and one reviewed neither. Two of the eight practices with a single suicide held independent reviews outside the study. CIRs were held 91215 days after the inquest.

Only 17 of a possible 52 GPs attended the CIR of a practice patient. The maximum number of GPs who could have attended a single review was eight, but the maximum number who actually attended was three. The number of practice members who attended a CIR ranged from one to six, with five reviews being conducted solely by the patient's GP. In contrast, two reviews included members from more than one practice.

\section{Were next of kin willing to contribute?}

Relatives of four patients (two spouses and three parents) agreed to discuss their relative's GP care with an independent interviewer (fig 2 and box 3). Three families felt there was nothing their GP or other health professional could have done to prevent the death, and that the responsibility lay with the individual concerned. Two of the deaths were viewed as 


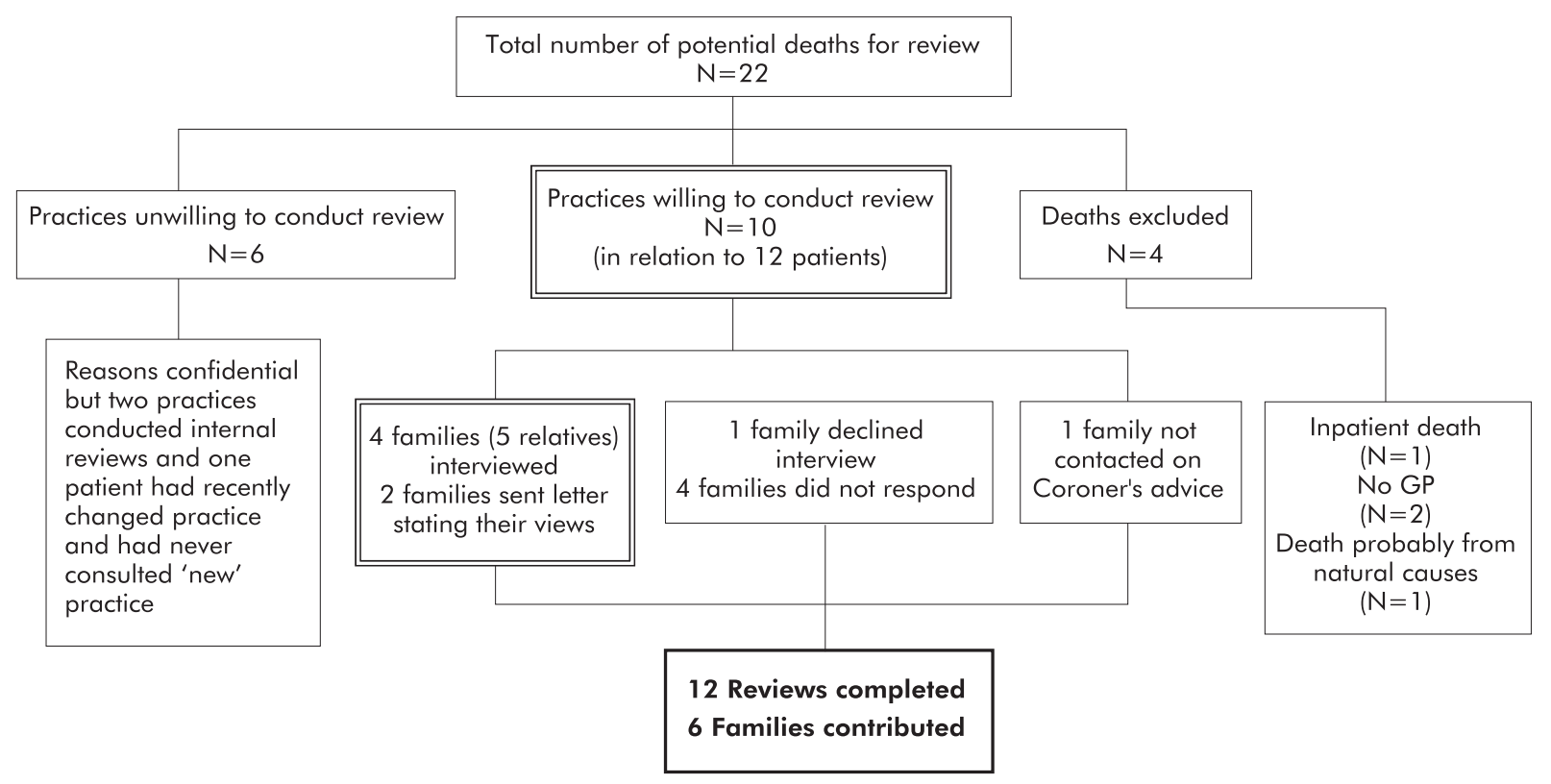

Figure 2 Response to requests to participate in critical incident reviews (CIRs) after patient suicide.

completely unexpected. Although the third case was due to an overdose of prescribed medication, the family had only praise for their relative's health care, particularly the consistently good support received from the GP, community psychiatric nurse (CPN), and psychiatrists. The fourth family identified specific issues they wanted to raise which they hoped might change practice and prevent other families having a similar experience. In criticising their relative's care, they cited poor communication between secondary and primary care, poor communication between GPs and parents

\section{Box 3 Summary of findings}

- Study period: 10 months

- Total number of eligible practices: 49

- Total number of eligible GPs: 190

- Total number of deaths eligible for CIR: 18 (registered with 13 practices)

- Total number of deaths ineligible for CIR: 4

- 1 in 4 practices had at least one suicide in the 10 month period

- 1 in 10 GPs had at least one patient suicide

- Outcome:

- No suicides in 36 practices

- 18 suicides in 13 practices

- 5 practices had 2 suicides

- 2 practices held CIRs on both patients

- 2 practices held a CIR on one patient

- 1 practice held no CIR on either patient

- 8 practices had 1 suicide

- 6 practices held a CIR

- 2 practices held independent internal reviews

- 6 families agreed to participate

- 5 relatives (of 4 suicides) were interviewed

- 2 relatives declined to be interviewed but expressed their views in a letter to GP of adult children who could usefully provide support, perceived poor communication between another professional and GP, and failure of their GP to refer their relative to a psychiatrist. They also criticised the lack of contact by their GP after the death.

Two of the families who declined to be interviewed expressed their satisfaction with the GP's care in unsolicited letters.

\section{Did CIRs lead to changes in practice?}

The most common issue identified as problematic was communication, both within the practice and with external agencies, and before and after the death.

\section{Internal changes}

The nature and quantity of prescribed drugs was identified as an issue, particularly when taken in fatal overdose. However, not all practices went on to implement changes in relation to this. A further issue was failures in record keeping where information had not been recorded in the patient's notes and previous case notes were not always available at the last consultation. This resulted in suggestions for improved record keeping. A GP's failure to contact relatives after a suicide had upset one family, and deficiencies in procedures for contacting relatives were recognised by some practices. Several practices decided to formalise their procedures for contacting relatives following a suicide after identifying the ad hoc way that this had been managed in the specific cases being reviewed. One practice also developed a risk assessment procedure to help them identify patients at risk.

\section{External changes}

Lack of communication between the Coroner's office and the practice was criticised by almost all GPs. All wanted to know the cause of death and felt that the inquest verdict should be the last entry in the patient's file as "the end of the story". Perceived poor communication between mental health professionals, other secondary care professionals, the police, and the practice was an issue for several practices since it was felt improved communication could have alerted the GP to potential risk factors before the death. 
Although it is difficult for individual practices to improve communication with external agencies, follow up of the issues raised at individual CIR meetings led to procedural changes that could potentially benefit all practices. Discussions with the Coroners' offices about the information that GPs would like resulted in new or amended procedures. Failure to inform a GP of a relevant contact with a secondary service had been addressed by one GP in preparation for the review and resulted in a change in procedure. The possibility of improving communication by the police to the practices in the event of a patient's arrest was explored by the CIR research team but aborted because of perceived data protection difficulties.

\section{Cost of CIRs}

The total cost of conducting the CIRs in this study, which each lasted about an hour, was estimated by summing the average hourly rate for the profession of each practice member who attended each CIR, based on data supplied by the NHS trust. The cost of facilitation (based on a consultant psychiatrist's rate) was similarly calculated.

On this basis, the estimated total cost of GP and primary care staff time was $£ 1070$. The estimated cost of individual reviews ranged from $£ 62$ to $£ 170$, depending on the number and professions of the staff attending. The cost of the time spent by GPs on the paperwork and in rearranging other commitments was estimated at $£ 400$ (average £35 per patient). The facilitator estimated that the setting up, preparing for, and facilitating each meeting took 2-2.5 hours. The additional cost of facilitation depended on the profession of the facilitator: in the case of a consultant psychiatrist the cost would be approximately $£ 165$ per review based on an hourly cost of $£ 66 .{ }^{14}$

\section{Did GPs and primary care team members value the review process?}

Each of the 26 practice members attending CIRs (16 GPs, six nurses and four administrative staff) was interviewed. One GP who attended two separate CIRs was interviewed twice. The key findings from the interviews are shown in box 4 .

\section{Barriers to CIRs}

Practice staff were asked about their concerns and expectations about the CIR prior to their participation. A number commented that they were uncertain what to expect. Some participants recommended that practices be provided with greater detail about the process as well as guidance on the type of information they could prepare in advance. These changes would not only help to ease anxiety but also allow for a more efficient use of time.

The majority of interviewees stated that they were hesitant to participate in the CIR because they feared that it would be a witch-hunt and that they would be blamed for their patient's death. For example, GP17 stated: "I think it's just that it feels rather... I think it does feel threatening doesn't it? So and so died, we are going to do a critical incident review kind of thing, it just sounds like we are going to find out, you know, I don't know, I honestly don't know how it's ... I don't know how it feels to other people but I think they probably do, I mean people do feel threatened by it you know this is sort of just a general idea that it's a finding out and a witch hunt or something. That might be a barrier."

Related to this fear was a concern that family members would unfairly take their grief and anger out on staff. Some stated that they would probably not have agreed to participate if they knew they had made a mistake. These fears were partially related to a fear of litigation. Many commented that they thought patients were becoming increasingly litigious and were concerned that documentation prepared for a CIR might be used against them in future legal action.

\section{Box $4 \mathrm{Key}$ issues from interviews}

- Barriers: fear of a witch-hunt; anxiety about raised expectations of ability of reviews to prevent suicides; a drain on time and resources.

- Benefits: opportunity for team building; improved communication and support; improved suicide risk assessment; informing future in-house reviews.

- Having an experienced facilitator known to the practice was identified as important, and the learning experience appeared greatest when the interval between inquest and review was short. No issues were identified that could have directly prevented a patient suicide.

With such anxiety in mind, participants thought that people generally would be reluctant to participate in CIRs and that, if they did take part, they may not be completely honest. There was also concern that the very fact of having a CIR would generate suspicion. GP7 described the tension she felt in desiring honest discussion but fearing litigation: "I don't know. I find that at the back of my brain there is always the fear when you're totally honest about something, that's the minute it'll get into the hands of $x$ 's solicitor. And they don't see things as a lot of the discussions, they would have a field day. And then you don't get any reasonable discussion moving forward. You just get the correct answer."

Most of the participants commented that the CIR imposed an additional burden on both their time and resources at a stage when they were already feeling very pressured. There was a feeling that more and more was being asked of GPs and practice staff generally, particularly with regard to clinical audit and governance. A few stated that, while they appreciated the provision of locums in this study, it did not sufficiently cover the time lost to CIRs. Furthermore, there were difficulties in finding a suitable time for everyone in the practice to attend, especially given the already high demands on time. These problems meant that it was difficult for staff to attend the CIR. GP9 described how increased and competing pressures made it difficult for him to find time for critical incident reviews: "But, but, but another negative thing, you know, about critical incident reviews, if you are doing lots of this type, because I think we have negative amounts of time, we are expected to do so much and more, every, every year and you know, fitting them in is difficult."

Given the barriers to attendance, each person interviewed was asked if everyone who should have attended the review did in fact attend. Most thought that this was the case, even where only the GP was present. A few GPs admitted that they did not advertise the meeting as widely as they could have done to colleagues because they did not know what to expect. This situation is illustrated by the interview with GP4: "I think to be fair, I think I was asked to attend and I, I think, it was said to me if anybody else had wanted to come, they were very welcome to come and I suspect I didn't sort of publicise the deal because obviously one wasn't ... I wasn't quite sure what it was ... it wasn't that I was worried about discussing it in anybody else, in anybody else's uh hearing. But I think I wasn't quite sure what, you know, what exactly it was about. So I think in future one would say, look come, come along, it's quite an interesting exercise and we might all learn a bit."

In three instances it was noted that it would have been very useful to have had the input of staff members, both non-GPs, who were absent. On one of these occasions the meeting was held outside the administrative staff member's working hours; in another the person chose not to attend because they had themselves been recently bereaved and felt unable to 
cope with the review of a suicide; and in a third the practice was short staffed.

Staff also identified some limitations and problems associated with CIRs. The most significant of these was a general consensus that CIRs would not really help to prevent most patient suicides. The participants regarded most suicides to be unpredictable and maintained that, if people really wanted to kill themselves, they would. There was a fear that CIRs might fuel an unrealistic expectation that GPs can prevent suicides so that, when they actually occurred, GPs would be held responsible. GP13 illustrates these concerns: "But there is a huge proportion of people that you give antidepressants to and no matter how hard you try to assess a suicide risk, you know, anyone who really wanted to kill themselves, if they have got any sense, they don't tell you. Where, whereas people who sit there and say 'oh yea I think about this and I think about that and I might do this and I might do that' yea, you, you try and explore it but at the end of the day that person is going to walk away from you after telling this and you are reliant upon what they have said to you and they can tell you a whole pack of lies and go and jump in front of a train, you know. But that's not my fault. That's their individual responsibility and how much they wish to impart, so that ... you know ... I don't control the situation but almost as if there, there is a feeling, by having these big forums and this formal stuff that I am responsible for that because I haven't foreseen it, but I don't have a crystal ball and know what they are going to do in the next 10-15 minutes. I can only try and assess a probability of risk."

\section{Benefits of CIRs}

Despite anticipating that the meeting would be a witch-hunt, the vast majority of practice members found the actual meeting to be unthreatening and beneficial in a number of ways. Most participants commented that it was very helpful to have an outside facilitator because of the new perspective and objectivity they provided. For example, Nurse 1 made the following comments: "Well, I think it helps because they're, well because they are coming in from outside, because they don't know us, they don't know anything about us and everything is, is, going to be ... well, I don't know really ... I mean they're coming from outside to deal with a situation that's something that's happened in the surgery. But they can view it objectively, can't they? And, and they, they've got the experience of other incidents as well. So they can pool all that together and hopefully they will be able to give us some ... um ... recommendations on how to deal with things in the future that we may not have realised that we should be doing or shouldn't be doing."

Additionally, since many of the participants already knew the facilitator, they were confident in his abilities. They felt they benefited from his experience as a psychiatrist as that enabled him to speak knowledgeably about suicide. The majority stated that they were able to speak freely, believing that confidentiality would be respected.

Where families provided input, practice members generally found this helpful and reassuring. Even in the one case where family members voiced concern, the GP generally regarded it positively because it led to improved communication. Indeed, it was felt that participation in the CIR contributed to enhanced communication with accident and emergency, the Coroner, and other practices. Some participants noted that the CIR also helped to improve communication within the practice, thus aiding team building. "Well, I think that when you sit down as a group, all of you-receptionists, doctors, nurses, outside people - and you hear what they know about the person, you know, it's good because you're learning from them as well as, as from the researchers. And I think it makes you stronger as a unit because, if something like this happens again you will know where to draw, draw your information, your strengths from each other." (Nurse 1)

Many stated that one of the key benefits was the knowledge they gained. Not only did they learn more about the particular event but about suicide more generally, including its associated risk factors. Furthermore, a number of practice members felt that their participation in the CIR taught them enough to run their own in-house review. More specifically, it provided them with a structure they could follow themselves.

Nearly all of the participants reported that one of the greatest benefits arising from the CIR was the fact that they were found to have done everything possible for the patient, and that warning signs had not been missed. As such, practice members felt absolved of guilt, relieved and reassured. Given the level of anxiety surrounding fear of blame and litigation, this is perhaps unsurprising. Even the opportunity to simply talk about the event was appreciated. A number of practice staff stated that the CIR aided them in dealing with their emotions-including guilt, anger, hurt and self-doubt-following the death of their patient. For example, GP10 recalled how the CIR helped to alleviate a sense of guilt: "One, I think it will make individuals feel a bit better about how they've proceeded in various situations or treated patients. Because you can feel a bit guilty, even if, even if you're obviously not, not at fault particularly. But you can feel a bit guilty. So it does, does help with those sorts of feelings."

\section{Future involvement and recommendations}

While most practice members were initially anxious and uncertain about attending the CIR meeting, their involvement generally created confidence to participate in future reviews, despite continued reservations in some situations of culpability and fear of litigation. It was thought that willingness to become involved in CIRs would increase through the experience of having been through one, word of mouth, and over time as people become more aware of the benefits.

Participants suggested that the reviews be held closer to the time of death, thus allowing for a fresher memory of the circumstances surrounding the event. Some doctors suggested that they be provided with information and training on suicide risk prediction. They thought that this might contribute to a greater reduction in suicides than a CIR.

\section{DISCUSSION}

This study differs from previous studies of CIR reviews in general practice $e^{71112}$ in that the views of relatives were sought and the confidential views of staff attending the meetings were obtained after the review. In this study the majority of practices were willing to hold CIRs. However, not all GPs in a practice attended and it was unusual for the wider primary health care team to attend. This may be explained by the considerable concerns GPs raised about CIRs prior to them taking place. GPs feared that CIRs might make them vulnerable to litigation and also viewed them as a drain on their time and resources. Given these concerns, it is perhaps puzzling why the majority of practices (if not staff members) did participate. This can be explained, at least in part, by the fact that these CIRs were conducted as part of a study with which practices were actively encouraged to participate and where additional resources (payment for locums) were offered. Additionally, the CIRs were facilitated by an external expert who was known to some of the practices.

Despite their initial reservations, study participants appeared to find the CIR process helpful in terms of reassuring them about their patient's death and improving their knowledge. One of the novel features of the CIR process in this study was to include the views of relatives. Only a small number of relatives were willing to be involved but, where they were, health professionals found this helpful and instructive. A number of changes in the procedures for 


\section{Key messages}

- The majority $(10 / 16)$ of practices with a patient suicide agreed to take part in a CIR but not all practice GPs or members of the primary health care team attended reviews.

- A few relatives were willing to contribute their views; health professionals found this helpful and instructive.

- Practice members were concerned about time and cost implications of CIRs and being blamed for patient deaths. This may mean practices are unwilling to initiate CIRs.

- CIRs can result in improved procedures and facilitate reflection on practice. Practice members valued the reassurance that warning signs had not been missed, as well as opportunities for team building, improved communication and support, learning about suicide risk factors, and guidance for future in-house reviews.

- The cost of external facilitation can be high, depending on the profession of the individual involved.

reviewing suicides and managing their aftermath were adopted as a result of CIRs. However, it is questionable whether any substantive measures were identified within primary care that would reduce the number of people committing suicide.

This study indicates that practices may be unwilling to initiate CIRs of patient suicides but that there are a number of benefits to such reviews taking place. CIRs can result in improved procedures, even if these are primarily at an individual practice level. Furthermore, they can facilitate reflection on practice in a difficult and potentially emotional area. After a patient suicide it is important to provide an opportunity for individual staff to deal with their feelings. However, the process involves staff in discussing the care they have provided to patients and perhaps identifying inadequacies in that care. Admitting to possible inadequacies of care, especially to colleagues, is an extremely stressful procedure for experienced health professionals exacerbated by fear of litigation or admission of personal failure. ${ }^{10}{ }^{11}$ Providing relatives with an opportunity to express their views is an important part of this process. Such feedback can provide practice staff with an important, often surprising, perspective on their patient's care and the way that relatives have experienced the event. Case discussion ideally requires an experienced facilitator to identify events in individual cases that have been critical, with a view to improving future care without attributing individual blame or self-criticism. ${ }^{10}$

In order to encourage practices to undertake and to benefit from CIRs, it seems likely that a system of external facilitation is necessary. Relatives should be given the opportunity to contribute their views to CIRs, but care is needed to ensure that they do not feel obliged to contribute and that there is appropriate assistance available should they become distressed. While relatives could be invited to participate by letter, an interview is probably the most appropriate way to gather the relevant information sensitively. This system of CIRs is costly and this may mean it is one with which practices may be reluctant to engage.

\section{ACKNOWLEDGEMENTS}

The authors thank HM Coroners for Southampton and the New Forest and for Central Hampshire and their staff for notifying them of the deaths and allowing access to the inquest files. They are also indebted to the GPs and their primary care team colleagues who were willing to participate in CIRs and to attend the post-CIR interviews.

\section{Authors' affiliations}

E King, K Kendall, R Wiles, C Gould, A Kendrick, University of Southampton, Southampton, UK

H Rosenvinge, University of Southampton and Consultant in Old Age Psychiatry, Hampshire Partnership NHS Trust, UK

This study was sponsored by HOPE (formerly Wessex Medical Trust).

There are no competing interests.

\section{REFERENCES}

1 World Health Organisation. WHO, MND, MBD/00.4. Geneva: World Health Organisation. Available at: http://www.who.int/entity/mental_health/ media/en/59.pdf.

2 World Health Organisation. Prevention of suicidal behaviours: a task for all. SUPRE-WHO, 2000. Available at: http://www.whosa.org/it/text5/ 14_suicide.html.

3 Little J. Staff response to inpatient and outpatient suicide: what happened and what did we do? Aust NZ J Psychiatry 1992;26:162-7.

4 Morgan H, Priest P. Suicide and other unexpected deaths among psychiatric inpatients: the Bristol confidential inquiry. Br J Psychiatry 1991;158:368-74.

5 Burgess P, Pirkis J, Morton J, et al. Lessons from a comprehensive clinical audit of users of psychiatric services who committed suicide. Psychiatr Serv 2000;51:1555-60.

6 National Health Service. National Service Framework: Standard Seven: Preventing suicide. London: NHS, 2001. Available at: http:// www.nelh.nhs.uk/nsf/mentalhealth/standard7.htm.

7 Berlin A, Spencer J, Bhopal R, et al. Audit of deaths in general practice: pilot study of the critical incident technique. Qual Health Care 1992;1:231-5.

8 Pringle M, Bradley C, Carmichael C, et al. Significant event auditing: a study of the feasibility and potential of case-based auditing in primary medical care. London: Royal College of General Practitioners, 1995.

9 Robinson L, Stacey R, Spencer J, et al. Use of facilitated case discussions for significant event auditing. BMJ 1995;311:315-8.

10 Robinson L. Facilitated case discussion as a method of multi-professional clinical audit. PhD/MD Thesis: University of Newcastle, 1999.

11 Redpath L, Stacey A, Pugh E, et al. Use of the critical incident technique in primary care in the audit of deaths by suicide. Qual Health Care 1997;6:25-8.

12 Pringle M. Significant event auditing. Scand J Primary Health Care 2000;18:200-2.

13 Glaser BG, Strauss AL. The discovery of grounded theory: strategies for qualitative research. Chicago: Aldine, 1967.

14 Personal Social Services Research Unit (PSSRU). Unit costs of health and social care. 2003. http://www.pssru.ac.uk/pdf/uc2003/uc2003_s14.pdf. 
APPENDIX 1 PRE-CRITICAL INCIDENT REVIEW QUESTIONNAIRE

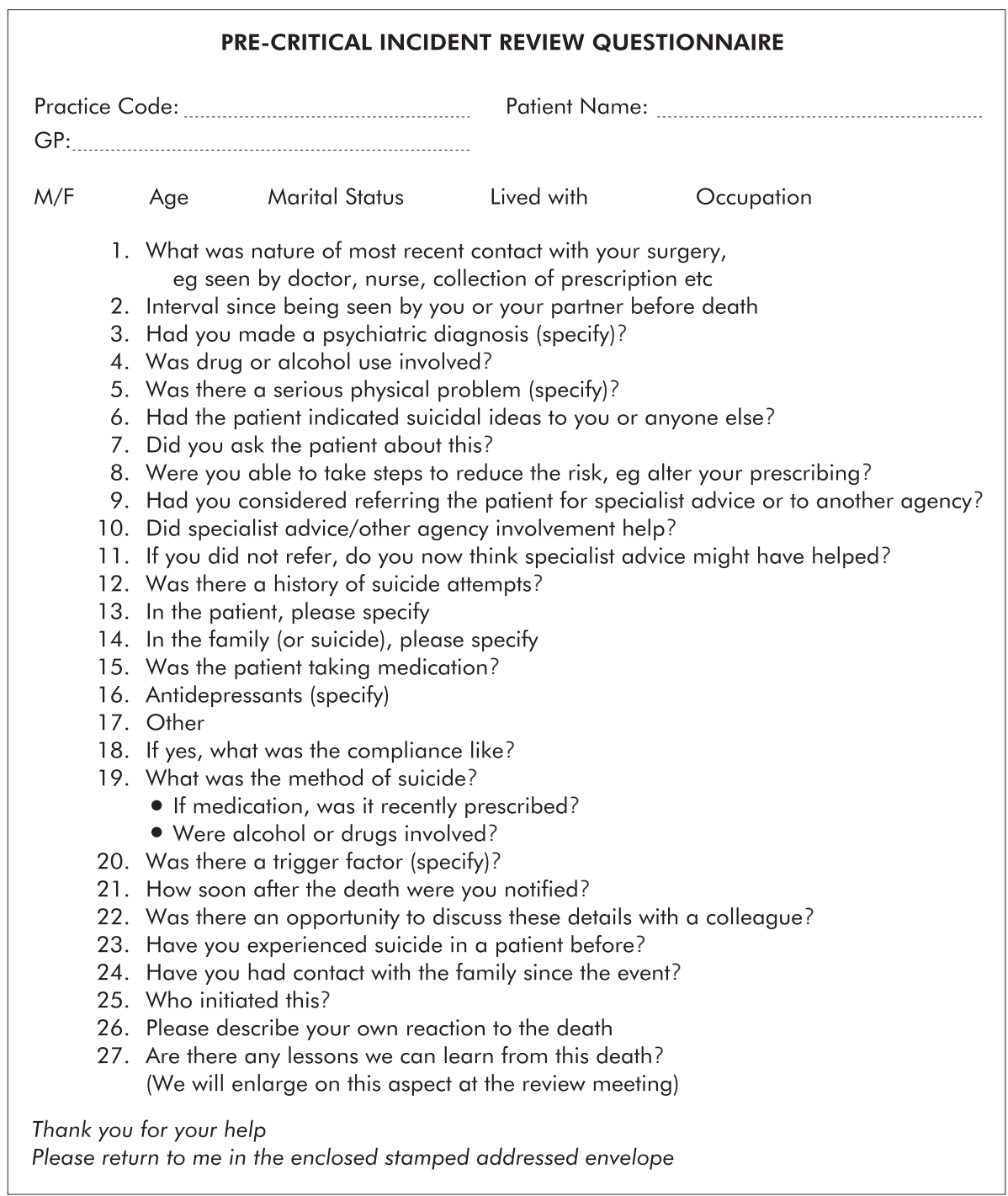

